\title{
Assessment of genetic potential and stress selection indices for important yield related traits in bread wheat ((Triticum aestivum L.)
}

\author{
Muhammad Ishaq ${ }^{1 *}$, Gulzar Ahmad ${ }^{1}$, Khilwat Afridi ${ }^{1}$, Murad Ali ${ }^{1}$, \\ Tanzim Ullah Khan ${ }^{1}$, Irfan Ahmad Shah ${ }^{1}$, Muhammad Khalid ${ }^{1}$, Nazir \\ Ahmad $^{2}$, Baber Iqbal ${ }^{1}$, Bilal Ahmad ${ }^{1}$, Muhammad Adeel Qureshi ${ }^{1}$, Amir \\ Saleem ${ }^{1}$, Muhammad Miraj ${ }^{3}$ and Kiramat Din $^{3}$ \\ 1. Cereal Crops Research Institute (CCRI), Pirsabak Nowshera Khyber Pakhtunkhwa-Pakistan \\ 2. Agricultural Research Institute, Tarnab Peshawar Khyber Pakhtunkhwa-Pakistan \\ 3. Department of Plant Breeding and Genetics, The University of Agriculture, Peshawar-Pakistan \\ *Corresponding author's email: ishaqaup@gmail.com \\ Citation \\ Muhammad Ishaq, Gulzar Ahmad, Khilwat Afridi, Murad Ali, Tanzim Ullah Khan, Irfan Ahmad Shah, Muhammad \\ Khalid, Nazir Ahmad, Baber Iqbal, Bilal Ahmad, Muhammad Adeel Qureshi, Amir Saleem, Muhammad Miraj and \\ Kiramat Din. Assessment of genetic potential and stress selection indices for important yield related traits in bread \\ wheat ((Triticum aestivum L.). Pure and Applied Biology. Vol. 7, Issue 3, pp1104-1114. \\ http://dx.doi.org/10.19045/bspab.2018.700129
}

Received: 31/01/2018 Revised: 01/08/2018

Accepted: 03/08/2018

Online First: 06/08/2018

\section{Abstract}

Current wheat improvement programs focuses on development of new high yielding, disease resistant, stable and climatic resilient genotypes. Late planting is one of the major abiotic stresses, seriously influencing wheat production. In the current study, twenty seven bread wheat genotypes along with one check cultivar (Pirsabak-2008) were evaluated independently under normal (optimal) and late (stress) planting conditions at Cereal Crops Research Institute (CCRI), Pirsabak Nowshera Khyber Pakhtunkhwa Pakistan during 2013-14. Analysis of variance revealed highly significant $(\mathrm{P} \leq 0.01)$ differences among the genotypes, sowing dates and genotype $\times$ sowing dates interactions effects for the studied traits. Generally, reduction in plant height ( 0.41 to $10.91 \%)$ and grain yield (0.36 to 53.35\%) was observed among the tested genotypes under late planting as compared to normal (optimal) planting. Least \% reduction in grain yield was recorded for genotypes BWL-23 (0.36\%), BWL-4(0.76\%) and BWL-16 (1.22\%) as compared to check (Pirsabak-2008). Eight stress selection indices i.e. Mean Productivity (MP), Tolerance (TOL), Geometric Mean Productivity (GMP), Harmonic mean (HM), Stress Selection Indices (SSI), Stress Tolerance Index (STI), Yield Index (YI) and Yield Stability Index (YSI) were determined for each genotype. Correlation analysis revealed that plant height and grain yield under both the planting conditions, had significant positive correlation with stress selection indices i.e. GM, HM, SSI and YI. These selection indices could be effective in identification of genotypes to late planting. Based on MP, GMP, HM, STI and YI genotypes i.e. Pirsabak-2008, BWL-23 and BWL-27 were found late planting stress tolerant and could be sown in both normal and late planting conditions.

Keywords: Abiotic stress; Genetic potential; Grain yield; Normal and late planting; Stress selection Indices; Tolerance; Variabilit 


\section{Introduction}

Wheat (Triticum aestivum L.) belongs to family poaceace and is one of the most important staple food of more than $36 \%$ of the world population. Its demand is increasing day by day due to ever-increasing population and its utilization in a variety of ways [1]. Its production has been greatly affected by various biotic and abiotic stresses. Among the abiotic stresses, late planting and sudden variable climatic changes (less rainfall, drought, heat stress at sowing time and increase in temperature at the terminal stages of crop) are the major threats and seriously influencing wheat production. Poor agronomic management practices i.e. unavailability of superior wheat genotypes for marginal environments, inefficient utilization of fertilizers and irrigation water etc are the key factors responsible for low wheat production. In Pakistan wheat being an important cereal crop its production is much better than the past but wheat yield is still behind compared to agricultural developed countries of the world. In Pakistan, late planting is among one of the major reason for low yield. Late planting adversely affects the growth, yield and quality of wheat. A delay of each day in sowing of wheat after 15th November onward decreases grain yield by $1 \%$. Late sowing of wheat can reduce yield by 30 to $40 \%$ [2]. Wheat cultivation at its proper time is thus of extreme importance to obtain high yield as proper sowing time accounts for $10 \%$ of grain variation [1]. In the current scenario of climatic change to feed the over increasing population there is dire need to develop new climatic resilient cultivars that produced higher yield under variable climatic conditions especially wheat planted late by farmers.

Exploration and identification of diverse climatic resilient wheat genotypes perform better under variable environmental condition (especially late planting or heat stress) is one of the main tasks of wheat breeders. To improve stress tolerance or identify stress tolerant lines in wheat several selection indices have been used by wheat breeders, when genotypes are evaluated under normal and stress conditions $[3,5]$. Tolerance (TOL) and mean productivity (MP), stress susceptibility index (SSI [6], geometric mean productivity (GMP) and stress tolerance index (STI [7] have all been employed under various conditions. In order to identify stable genotype under stress and non-stress conditions indices like yield index (YI), yield stability index (YSI) were proposed by $[8,9]$. Keeping in view the importance of improving wheat production under the current variable climatic conditions this research was undertaken with the objectives to: (i) Screen and identify potential lines that perform better under normal and late planting conditions. (ii) Identify late planting stress tolerant genotypes of wheat based on stress selection indices. (iii) Utilization of the germplasm in future breeding programs especially breeding wheat for late planting.

\section{Materials and methods}

\section{Experimental site and genetic material}

To explore the genetic potential and asses stress tolerant genotypes, the study was carried out at Cereal Crops Research Institute (CCRI), Pirsabak Nowshera Khyber Pakhtunkhwa-Pakistan during crop season of 2013-2014. The experimental site is located at elevation of 288 meters $(945 \mathrm{Ft})$ and located on the intersection of longitude $740 \mathrm{E}$ and latitude $320 \mathrm{~N}$ and having silt loam with soil $\mathrm{pH}$ of 7.8. Breeding material comprised a set of 28 wheat genotypes (27 lines and one check cultivar). Details of the genotypes used in the study are presented in (Table 1). These genotypes were evaluated as independent experiments under normal and late planting conditions. Both experiments were conducted in Randomized Complete Block Design with three replications. The normal experiment was planted on November 15, 2013 while, late experiment was planted on December 15, 2013. Each genotype was planted in 5 rows 
plot $^{-1}$, with row length of 5 meter and row-torow spacing of $30 \mathrm{~cm}$ under each environment. In both the experiments standard cultural practices were adopted including fertilizers and other crop husbandry practices throughout the growing season. Data was recorded on plant height $(\mathrm{cm})$ and grain yield $\left(\mathrm{kg} \mathrm{ha}^{-1}\right)$ in both the experiments on all the genotypes. Data were analyzed across the two environments using mixed effect model to ascertain genotype $\times$ environment interaction effect for each trait. [10].

Table 1. List of the 27 bread wheat advanced lines received from CIMMYT-Mexico and local check used in the study

\begin{tabular}{|c|c|c|}
\hline S. No. & Line code & Parentage/Pedigree \\
\hline 1 & BWL-1 & PBW343*2/KUKUNA// PBW343*2/KUKUNA \\
\hline 2 & BWL-2 & OUT//PRL/2*PASTOR \\
\hline 3 & BWL-3 & SW89.5277/BORL95//SKAUZ/3/PRL/2*PASTOR/4/ \\
\hline 4 & BWL-4 & WAXWING*2// INQLAB 91*2/KUKUNA/3/WBLL1*2/ \\
\hline 5 & BWL-5 & ROLFO7*2/KIRITATI \\
\hline 6 & BWL-6 & HUW234+LR34/PRINIA*2//WHEAR \\
\hline 7 & BWL-7 & CAL/NH//H567.71/3/SERI/4/CAL/NH//H567.71/5/ \\
\hline 8 & BWL-8 & SOKOLL//SUNCO/2* PASTOR \\
\hline 9 & BWL-9 & SOKOLL/PRL/2*PASTOR \\
\hline 10 & BWL-10 & CHEN/AEGILOPS SQUARROSA (TAUS)//BCN/3/BAV92/... \\
\hline 11 & BWL-11 & CROC_1/AE. SQUARROSA (205)/KAUZ/3/PASTOR... \\
\hline 12 & BWL-12 & $\begin{array}{c}\text { KAUZ//ALTAR84/AOS/3/MILAN/KAUZ/4/HUITES/7/CAL/NH//H567. } \\
\text { 71/3/SERI/4/CAL/NH//H567.71/5/2*KAUZ/6/PASTOR }\end{array}$ \\
\hline 13 & BWL-13 & $\begin{array}{c}\text { FRET2*2/4/SNI/TRAP\#1/3/KAUZ*2/TRAP//KAUZ/5/PARUS/6/FRET2 } \\
* 2 / \text { KUKUNA }\end{array}$ \\
\hline 14 & BWL-14 & ATTILA*2/PBW65*2/4/BOW/NKT//CBRD/3/CBRD \\
\hline 15 & BWL-15 & $\begin{array}{l}\text { WBLL1*2/BRAMBLING/5/BABAX/LR42//BABAX*2/4/SNI/TRAP\#1/ } \\
\text { 3/KAUZ*2/TRAP//KAUZ }\end{array}$ \\
\hline 16 & BWL-16 & W15.92/4/PASTOR//HXL7573/2*BAU/3/WBLL1 \\
\hline 17 & BWL-17 & FRET2*2/4/SNI/TRAP\#1/3/KAUZ*2/TRAP//KAUZ/5/ONIX \\
\hline 18 & BWL-18 & ONIX/ROLF07 \\
\hline 19 & BWL-19 & $\begin{array}{c}\text { CNO79//PF70354/MUS/3/PASTOR/4/BAV92/5/FRET2/KUKUNA//FRE } \\
\text { T2/6/MILAN/KAUZ//PRINIA/3/BAV92 }\end{array}$ \\
\hline 20 & BWL-20 & CUNNINGHAM/4/SNI/TRAP\#1/3/KAUZ*2/TRAP//KAUZ \\
\hline 21 & BWL-21 & SOKOLL*2/4/CHEN/AEGILOPSSQUARROSA (TAUS)//FCT/3/STAR \\
\hline 22 & BWL-22 & MTRWA92.161/PRINIA/5/SERI*3//RL6010/4*YR/3/PASTOR/4/ \\
\hline 23 & BWL-23 & MTRWA92.161/PRINIA/5/SERI*3//RL6010/4*YR/3/PASTOR/4/ \\
\hline 24 & BWL-24 & BERKUT/EXCALIBUR \\
\hline 25 & BWL-25 & QUAI \\
\hline 26 & BWL-26 & PJN/BOW//OPATA/3/HXL7573/2*BAU \\
\hline 27 & BWL-27 & $\begin{array}{l}\text { TRCH/5/REH/HARE//2*BCN/3/CROC_1/AE.SQUARROSA } \\
(213) / / \text { PGO/4/HUITES }\end{array}$ \\
\hline 28 & Check (Pirsabak-2008) & Local Check (Pirsabak-2008) \\
\hline
\end{tabular}

\section{Stress selection Indices}

The experimental material planted in November (normal) and in December (late sown) was considered as non-stress and stress environment, respectively to determine the following selection indices. Let $\mathrm{YN}=$ Yield of a given genotype in normal planting (non- stress) environment; $\mathrm{YS}=$ Yield of a given genotype in stress (late planting) environment; $\overline{Y n}=$ Mean yield under nonstress environment and $\overline{Y S}=$ Mean yield under stress environment. The various stress tolerance indices of wheat genotypes for the 
studied traits are determined by the following formulas;

1. Tolerance Index (TOL) [11]. Tol = $\mathrm{YN}-\mathrm{YS}$

2. Mean Productivity (MP) [11]. MP = $\frac{\mathrm{YN}+\mathrm{YS}}{2}$

3. Harmonic Mean (HM) [12]. HM = $\frac{2(\mathrm{YN} \times \mathrm{YS})}{\mathrm{YN}+\mathrm{YS}}$

4. Stress Susceptibility Index (SSI) [8].

$\mathrm{SSI}=1-\frac{\left(\frac{Y S}{Y N}\right)}{1-\left(\frac{\overline{Y S}}{\overline{Y n}}\right)}$

5. Geometric Mean Productivity (GMP) [7]. GMP $=\sqrt{\mathrm{YN} \times \mathrm{YS}}$

6. Stress Tolerance Index (STI) [7]. $\mathrm{STI}=(\mathrm{YN} \times \mathrm{YS}) /(\overline{Y n}) 2$

7. Yield Index (YI) $[8]$. YI $=Y S / \overline{Y S}$

8. Yield stability index (YSI) [9]. YSI = YS/YN

Table 2. Mean squares for plant height and grain yield of wheat genotypes evaluated under normal and late planting

\begin{tabular}{|c|c|c|c|}
\hline Source of variation & df & Plant height & Grain yield \\
\hline Replications & 2 & $68.077^{*}$ & $14827.4^{\text {NS }}$ \\
\hline Genotypes & 27 & $104.475^{* *}$ & $2004438^{* *}$ \\
\hline Sowing dates & 1 & $546.482^{* *}$ & $15530000^{* *}$ \\
\hline Genotypes $\times$ Sowing dates & 27 & $20.445^{* *}$ & $612173^{* *}$ \\
\hline Error & 110 & 8.386 & 165619 \\
\hline CV $(\%)$ & - & 2.81 & 10.09 \\
\hline
\end{tabular}

$*$ and $* *=$ Significant at $5 \%$ and $1 \%$ level of probability, respectively NS = Non-significant

$\mathrm{CV}=$ Coefficient of variation

Mean performance and stress selection indices

\section{Plant height}

Mean performance and stress selection indices for plant height are presented in (Table 3). Plant height under normal and late planting varied from 97.0 to $112 \mathrm{~cm}$ and 95 to 107.7 $\mathrm{cm}$, respectively. Maximum plant height under normal and late planting was observed for BWL-27. Under late planting, minimum

\section{Correlation analysis}

Simple correlation among the stress selection indices and the studied traits under normal and late planting conditions was carried out using statistical software (Statistix-8.1).

\section{Results and Discussion}

\section{Analysis of genotypic variation}

Analysis of variance revealed highly significant $(\mathrm{P} \leq 0.01)$ differences among the genotypes, sowing/planting dates and genotypes $\times$ sowing dates for plant height and grain yield (Table 2). Our results are in agreement with those of $[13,14]$ who found significant variation among the genotypes for plant height and grain yield under optimal and late planting. Significance of genotypes $x$ sowing dates interaction indicated that these genotypes performed differently under normal and late planting conditions and sufficient diversity existed among the evaluated germplasm under both the planting environments. Significant genotypic variations for the studied traits among the two planting environments have also been reported by $[15,17]$. 
(8.67) were least tolerant as more reduction in plant height was observed. For plant height mean productivity (MP) ranged from 96.00 to $110.00 \mathrm{~cm}$. Based on mean productivity (MP) for plant height genotype BWL-27 (110.0 $\mathrm{cm}), \quad$ BWL-26 (107.83 cm) BWL-9 $(107.67 \mathrm{~cm})$ were most tolerant while, BWL-8 $(96.0 \mathrm{~cm})$ was least tolerant. Based on harmonic mean, BWL-27 $(109.95 \mathrm{~cm})$ and BWL-26 $(107.72 \mathrm{~cm})$ was found highly stress tolerant whereas, genotype BWL-8 (95.99 $\mathrm{cm})$ was found least stress tolerant. Stress Susceptibility Index (SSI) which determines reduction in performance of a genotype under stress environment. If SSI < 1 , it indicated that genotypes are highly stress tolerant and vice versa. Based on SSI genotypes, BWL13(0.07), Pirsabak-2005 (0.15), BWL-3 (0.22) and BWL-16 (0.22) were highly stressed tolerant. Geometric mean productivity (GMP) varied from 95.99 to $109.98 \mathrm{~cm}$. GMP, identified BWL$27(109.98 \mathrm{~cm})$ and BWL-26 $(107.78 \mathrm{~cm})$ most tolerant whereas, least tolerant genotype was BWL-8 (95.99 cm). STI (Stress Tolerance Index) is used to identify genotype performed better under normal and late planting (stress) conditions. The larger the STI value, the greater is stress tolerance and yield potential. Based on STI, BWL-27(1.19) and BWL26(1.15) were most tolerated stress genotypes while; BWL-8 (0.91) was least tolerant among the genotypes. Yield index (YI) determine performance of genotypes under stress environment. YI ranked genotypes BWL-27 (1.07), BWL-6 (1.05), BWL-10 (1.05) as most tolerant. However, BWL-1(0.91) and BWL$14 \&$ BWL-15(0.94) were least tolerant genotypes. Yield Stability Index (YSI) determines performance of genotype under stress conditions. According to YSI, best genotypes were BWL-13(1.0), BWL-3, BWL-16, BWL-25 and Pirsabak-2008 whereas; BWL-12 (0.92) and BWL-5(0.93) were least tolerant. For plant height \% reduction range from 0.41 to $10.91 \%$ among the genotypes and BWL-13 (0.41\%), BWL-16 (1\%), BWL-3 and BWL-25 (1.24\%) were found more tolerated genotypes as less reduction in plant height was observed (Figure 1). Decrease in plant height was due to genotypes planted late, which decreases the normal growth period and vegetative growth. Our results are supported by $[18,19]$ who found reduction in plant height while planting wheat genotypes late.

\section{Grain yield}

Data pertaining to mean grain yield and stress selection indices are presented in (Table 4). Under normal planting, grain yield varied from 3123.1 to $5644.1 \mathrm{~kg} \mathrm{ha}^{-1}$. Maximum and minimum grain yield under normal planting was recorded for Pirsabak-2008 and BWL-25, respectively. Grain yield under late planting ranged from 2744.4 (BWL-5) to $5000 \mathrm{~kg} \mathrm{ha}^{-1}$ (Pirsabak-2008). TOL varied from 17.22 to $1464.07 \mathrm{~kg} \mathrm{ha}^{-1}$. TOL index showed that genotypes BWL-23 (17.22 kg ha-1), BWL-4 (35.19 $\left.\mathrm{kg} \mathrm{ha}^{-1}\right)$, BWL-16 (37.96 kg ha-1) were most tolerant as least reduction in grain yield was observed whereas, genotypes, BWL-5 (1464.07 kg ha-1), BWL-1 (1443.33 kg ha') and BWL-10 (1385.0 kg ha-1) were least tolerant as more reduction in grain yield was observed. Based on mean productivity (MP) for grain yield Pirsabak-2008 $\left(5322.04 \mathrm{~kg} \mathrm{ha}^{-}\right.$ $\left.{ }^{1}\right)$, BWL-23 (4766.94 kg ha-1), BWL-27 (4752.04 $\mathrm{kg} \mathrm{ha}^{-1}$ ) were found most tolerant whereas, BWL-25 (268.11 kg ha $\left.{ }^{-1}\right)$, BWL-16 $\left(3143.98 \mathrm{~kg} \mathrm{ha}^{-1}\right)$ and BWL-13 (3172.41 kg $\mathrm{ha}^{-1}$ ) were least tolerant. Based on harmonic mean Pirsabak-2008 (5302.55 kg ha $\left.{ }^{-1}\right)$, BWL12 (4944.26 kg ha-1) BWL-23 (4766.94 kg ha1) were found most tolerant whereas, genotypes BWL-23 (2608.17 kg ha-1), BWL$16\left(3143.87 \mathrm{~kg} \mathrm{ha}^{-1}\right)$ and BWL-13(3172.16 kg $\left.\mathrm{ha}^{-1}\right)$ ) were found least stress tolerant.

Under stress environment, reduction in performance or grain yield was also determined through stress susceptibility index (SSI). If SSI for a genotype is less than one it clearly indicated that the said genotype is 
highly stress tolerant to late planting and vice versa. Based on SSI genotypes BWL23(0.08), BWL-04 (0.16) and BWL-16 (0.26) were more resistant to stress condition While, BWL-7 (6.29), BWL-25 (6.22), BWL-10 (6.43) and BWL-5 (7.64) were least tolerant. Genotypes based on GMP, Pirsabak-2008 $\left(5312.28 \mathrm{~kg} \mathrm{ha}^{-1}\right)$ and BWL-12 (4962.63 kg $\mathrm{ha}^{-1}$ ), BWL-23 (4766.94 kg ha-1), BWL-27 $\left(4726.03 \mathrm{~kg} \mathrm{ha}^{-1}\right)$ were most tolerant however, least tolerant genotypes were BWL-25 (2644.39 kg ha-1), BWL-16 (3143.92 kg ha-1), BWL-13 (3172.28 $\left.\mathrm{kg} \mathrm{ha}^{-1}\right)$ were least tolerant. STI (Stress tolerance index) is used to identify genotype performed better under normal and late planting (stress). Based on STI, Pirsabak2008 (2.11), BWL-12 (1.84), BWL-23 (1.70) and BWL-27 (1.67) were most tolerated stress genotypes while; BWL-25 (0.52), BWL-16 (0.74) and BWL-3 (0.92) were least tolerant among the genotypes. Yield index (YI) determine performance of genotypes under stress environment. YI ranked genotype Pirsabak-2008 (1.37), BWL-23 (1.30), BWL4 (1.27), BWL-12 (1.24) and BWL-26 (1.16) most tolerant. However, BWL-25 (0.61) and BWL-13 (0.85) \& BWL-13 (0.86) were least tolerant. Yield stability index (YSI) determine performance of genotype under stress. According to YSI, desirable genotypes were BWL-23 (1.00), BWL-4 (0.99), BWL-16 (0.99), BWL-13 (0.99) whereas; genotypes (BWL-1, BWL-7, BWL-10 (0.71) and BWL25 (0.72) were least tolerant. MP, GMP and STI indices are preferred for practical uses also have been reported by [20, 21]. MP, GMP and STI had similar ability to differentiate genotypes for drought sensitiveness and tolerance was reported by [5]. Grain yield reduction was observed under late planting stress environment as compared to normal (optimal) sowing conditions. Least \% reduction in grain yield was recorded for genotypes, BWL-23 (0.36\%), BWL$4(0.76 \%)$, BWL-16 (1.22\%) and BWL-13 $(1.78 \%)$ and were found tolerant to late planting stress as compared to check (Pirsabak-2008) (Figure 2). Reduction in grain yield $(33 \%)$ due to late planting was also reported by [1]. Our results are in agreement with [18, 19] who reported grain yield reduction as a result of late planting. Considerable reduction in grain yield due to late sowing were also reported by $[23,24]$ in wheat.

\section{Correlation analysis}

Correlation analysis depicted that plant height under normal planting has strong positive correlation with GM $\left(\mathrm{r}=0.96^{* *}\right)$, TOL $(\mathrm{r}$ $\left.=0.55^{* *}\right), \mathrm{MP}\left(\mathrm{r}=0.96^{* *}\right), \mathrm{HM}\left(\mathrm{r}=0.95^{* *}\right)$, SSI $\left(\mathrm{r}=0.48^{* *}\right)$ and YI $\left(\mathrm{r}=0.81^{* *}\right)$ and negative correlation with YSI $\left(\mathrm{r}=-0.46^{* *}\right)$. Similarly under late planting positive correlation of plant height with stress selection indices i.e. GM $(\mathrm{r}=0.94 * *)$, MP $(\mathrm{r}$ $=0.94 * *), \mathrm{HM}(\mathrm{r}=0.95 * *), \mathrm{STI}(\mathrm{r}=0.94 * *)$ and YI $\left(\mathrm{r}=1.00^{* *}\right)$ and negative correlation with TOL $(r=-0.05)$ SSI $(r=-0.13)$ (Table 5). Correlation analysis showed that grain yield under normal planting has strong positive correlation with GM $(\mathrm{r}=0.92 * *)$, TOL $(\mathrm{r}$ $\left.=0.46^{* *}\right), \mathrm{MP}\left(\mathrm{r}=0.93^{* *}\right), \mathrm{HM}\left(\mathrm{r}=0.91^{* *}\right)$ and YI $\left(\mathrm{r}=0.81^{* *}\right)$ and negative correlation with YSI $(r=-0.29)$. Similarly under late planting positive correlation grain yield with stress selection indices i.e. $\mathrm{GM}(\mathrm{r}=0.94 * *)$, MP ( $\left.\mathrm{r}=0.92^{* *}\right), \mathrm{HM}\left(\mathrm{r}=0.95^{* *}\right)$, STI ( $\mathrm{r}$ $=0.94 * *)$, YSI $(\mathrm{r}=-0.44 * *)$ and YI $(\mathrm{r}=$ $1.00 * *)$ significant negative correlation SSI ( $\mathrm{r}$ $=-0.45)$ (Table 5). Our results are supported by $[5,21,22]$ who reported that GMP, MP and STI had positive correlation with yield under stress. We found perfect positive correlation between YI and grain yield under late sowing condition (YS) indicated its suitability for selecting tolerant genotypes under late sowing condition. This result is in agreement with the findings of [3]. 
Table 3. Mean and stress selection indices for plant height of wheat genotypes evaluated under normal (YN) and late planting (YS) conditions

\begin{tabular}{|c|c|c|c|c|c|c|c|c|c|c|}
\hline Genotypes & $\mathbf{Y N}$ & $\mathbf{Y S}$ & TOL & MP & HM & SSI & GMP & STI & YI & YSI \\
\hline BWL-1 & 101.7 & 91.7 & 10.00 & 96.67 & 96.41 & 2.30 & 96.54 & 0.92 & 0.91 & 0.90 \\
\hline BWL-2 & 101.7 & 99.7 & 2.00 & 100.67 & 100.66 & 0.43 & 100.66 & 1.00 & 0.99 & 0.98 \\
\hline BWL-3 & 97.7 & 96.7 & 1.00 & 97.17 & 97.16 & 0.22 & 97.17 & 0.93 & 0.96 & 0.99 \\
\hline BWL-4 & 107.7 & 105.0 & 2.67 & 106.33 & 106.32 & 0.54 & 106.32 & 1.11 & 1.04 & 0.98 \\
\hline BWL-5 & 109.3 & 102.0 & 7.33 & 105.67 & 105.54 & 1.47 & 105.60 & 1.10 & 1.01 & 0.93 \\
\hline BWL-6 & 109.0 & 105.7 & 3.33 & 107.33 & 107.31 & 0.67 & 107.32 & 1.14 & 1.05 & 0.97 \\
\hline BWL-7 & 111.3 & 100.7 & 10.67 & 106.00 & 105.73 & 2.10 & 105.87 & 1.10 & 1.00 & 0.90 \\
\hline BWL-8 & 97.0 & 95.0 & 2.00 & 96.00 & 95.99 & 0.45 & 95.99 & 0.91 & 0.94 & 0.98 \\
\hline BWL-9 & 108.0 & 103.3 & 4.67 & 105.67 & 105.62 & 0.95 & 105.64 & 1.10 & 1.03 & 0.96 \\
\hline BWL-10 & 111.7 & 105.3 & 6.33 & 108.50 & 108.41 & 1.25 & 108.45 & 1.16 & 1.05 & 0.94 \\
\hline BWL-11 & 103.7 & 96.7 & 7.00 & 100.17 & 100.04 & 1.48 & 100.11 & 0.99 & 0.96 & 0.93 \\
\hline BWL-12 & 108.7 & 100.0 & 8.67 & 104.33 & 104.15 & 1.75 & 104.24 & 1.07 & 0.99 & 0.92 \\
\hline BWL-13 & 98.7 & 98.3 & 0.33 & 98.50 & 98.50 & 0.07 & 98.50 & 0.96 & 0.98 & 1.00 \\
\hline BWL-14 & 100.7 & 95.0 & 5.67 & 97.83 & 97.75 & 1.24 & 97.79 & 0.94 & 0.94 & 0.94 \\
\hline BWL-15 & 100.0 & 95.0 & 5.00 & 97.50 & 97.44 & 1.10 & 97.47 & 0.94 & 0.94 & 0.95 \\
\hline BWL-16 & 101.0 & 100.0 & 1.00 & 100.50 & 100.50 & 0.22 & 100.50 & 1.00 & 0.99 & 0.99 \\
\hline BWL-17 & 111.7 & 103.7 & 8.00 & 107.67 & 107.52 & 1.57 & 107.59 & 1.14 & 1.03 & 0.93 \\
\hline BWL-18 & 105.0 & 100.0 & 5.00 & 102.50 & 102.44 & 1.05 & 102.47 & 1.04 & 0.99 & 0.95 \\
\hline BWL-19 & 107.3 & 104.0 & 3.33 & 105.67 & 105.64 & 0.68 & 105.65 & 1.10 & 1.03 & 0.97 \\
\hline BWL-20 & 102.0 & 99.0 & 3.00 & 100.50 & 100.48 & 0.65 & 100.49 & 1.00 & 0.98 & 0.97 \\
\hline BWL-21 & 106.0 & 101.7 & 4.33 & 103.83 & 103.79 & 0.90 & 103.81 & 1.06 & 1.01 & 0.96 \\
\hline BWL-22 & 108.0 & 103.3 & 4.67 & 105.67 & 105.62 & 0.95 & 105.64 & 1.10 & 1.03 & 0.96 \\
\hline BWL-23 & 107.0 & 104.7 & 2.33 & 105.83 & 105.82 & 0.48 & 105.83 & 1.10 & 1.04 & 0.98 \\
\hline BWL-24 & 104.7 & 100.7 & 4.00 & 102.67 & 102.63 & 0.84 & 102.65 & 1.04 & 1.00 & 0.96 \\
\hline BWL-25 & 106.0 & 104.7 & 1.33 & 105.33 & 105.33 & 0.28 & 105.33 & 1.09 & 1.04 & 0.99 \\
\hline BWL-26 & 111.3 & 104.3 & 7.00 & 107.83 & 107.72 & 1.38 & 107.78 & 1.15 & 1.04 & 0.94 \\
\hline BWL-27 & 112.3 & 107.7 & 4.67 & 110.00 & 109.95 & 0.91 & 109.98 & 1.19 & 1.07 & 0.96 \\
\hline Check (Pirsabak-2008) & 97.0 & 96.3 & 0.67 & 96.67 & 96.67 & 0.15 & 96.67 & 0.92 & 0.96 & 0.99 \\
\hline
\end{tabular}


Table 4. Mean and stress selection indices for grain yield of wheat genotypes evaluated under normal (YN) and late planting (YS) condition

\begin{tabular}{|c|c|c|c|c|c|c|c|c|c|c|}
\hline Genotypes & $\mathbf{Y N}$ & $\mathbf{Y S}$ & TOL & MP & HM & SSI & GMP & STI & YI & YSI \\
\hline BWL-1 & 5060.0 & 3616.7 & 1443.33 & 4338.33 & 4218.29 & 1.76 & 4277.89 & 1.37 & 0.99 & 0.71 \\
\hline BWL-2 & 4813.0 & 3488.9 & 1324.07 & 4150.93 & 4045.34 & 6.04 & 4097.79 & 1.26 & 0.95 & 0.72 \\
\hline BWL-3 & 3699.3 & 3327.8 & 371.48 & 3513.52 & 3503.70 & 2.21 & 3508.61 & 0.92 & 0.91 & 0.90 \\
\hline BWL-4 & 4685.2 & 4650.0 & 35.19 & 4667.59 & 4667.53 & 0.16 & 4667.56 & 1.63 & 1.27 & 0.99 \\
\hline BWL-5 & 4208.5 & 2744.4 & 1464.07 & 3476.48 & 3322.34 & 7.64 & 3398.54 & 0.86 & 0.75 & 0.65 \\
\hline BWL-6 & 4200.0 & 3175.0 & 1025.00 & 3687.50 & 3616.27 & 5.36 & 3651.71 & 1.00 & 0.87 & 0.76 \\
\hline BWL-7 & 4734.1 & 3377.8 & 1356.30 & 4055.93 & 3942.54 & 6.29 & 3998.83 & 1.20 & 0.92 & 0.71 \\
\hline BWL-8 & 4273.0 & 3480.6 & 792.41 & 3876.76 & 3836.27 & 4.07 & 3856.46 & 1.11 & 0.95 & 0.81 \\
\hline BWL-9 & 4178.5 & 3366.7 & 811.85 & 3772.59 & 3728.92 & 4.27 & 3750.69 & 1.05 & 0.92 & 0.81 \\
\hline BWL-10 & 4732.2 & 3347.2 & 1385.00 & 4039.72 & 3921.01 & 6.43 & 3979.92 & 1.19 & 0.92 & 0.71 \\
\hline BWL-11 & 4334.8 & 3719.4 & 615.37 & 4027.13 & 4003.62 & 3.12 & 4015.36 & 1.21 & 1.02 & 0.86 \\
\hline BWL-12 & 5413.3 & 4550.0 & 863.33 & 4981.67 & 4944.26 & 3.50 & 4962.93 & 1.84 & 1.24 & 0.84 \\
\hline BWL-13 & 3200.4 & 3144.4 & 55.93 & 3172.41 & 3172.16 & 0.38 & 3172.28 & 0.75 & 0.86 & 0.98 \\
\hline BWL-14 & 3721.9 & 3386.1 & 335.74 & 3553.98 & 3546.05 & 1.98 & 3550.01 & 0.94 & 0.93 & 0.91 \\
\hline BWL-15 & 4499.6 & 3894.4 & 605.19 & 4197.04 & 4175.22 & 2.95 & 4186.11 & 1.31 & 1.07 & 0.87 \\
\hline BWL-16 & 3163.0 & 3125.0 & 37.96 & 3143.98 & 3143.87 & 0.26 & 3143.92 & 0.74 & 0.85 & 0.99 \\
\hline BWL-17 & 4377.0 & 3272.2 & 1104.81 & 3824.63 & 3744.84 & 5.54 & 3784.53 & 1.07 & 0.90 & 0.75 \\
\hline BWL-18 & 4243.7 & 3686.1 & 557.59 & 3964.91 & 3945.30 & 2.89 & 3955.09 & 1.17 & 1.01 & 0.87 \\
\hline BWL-19 & 4397.8 & 3986.1 & 411.67 & 4191.94 & 4181.84 & 2.06 & 4186.89 & 1.31 & 1.09 & 0.91 \\
\hline BWL-20 & 3871.5 & 3769.4 & 102.04 & 3820.46 & 3819.78 & 0.58 & 3820.12 & 1.09 & 1.03 & 0.97 \\
\hline BWL-21 & 4677.8 & 3688.9 & 988.89 & 4183.33 & 4124.89 & 4.64 & 4154.01 & 1.29 & 1.01 & 0.79 \\
\hline BWL-22 & 5049.3 & 4063.9 & 985.37 & 4556.57 & 4503.30 & 4.29 & 4529.86 & 1.54 & 1.11 & 0.80 \\
\hline BWL-23 & 4775.6 & 4758.3 & 17.22 & 4766.94 & 4766.93 & 0.08 & 4766.94 & 1.70 & 1.30 & 1.00 \\
\hline BWL-24 & 3675.2 & 3533.3 & 141.85 & 3604.26 & 3602.86 & 0.85 & 3603.56 & 0.97 & 0.97 & 0.96 \\
\hline BWL-25 & 3123.3 & 2238.9 & 884.44 & 2681.11 & 2608.17 & 6.22 & 2644.39 & 0.52 & 0.61 & 0.72 \\
\hline BWL-26 & 4225.2 & 3713.9 & 511.30 & 3969.54 & 3953.07 & 2.66 & 3961.30 & 1.17 & 1.02 & 0.88 \\
\hline BWL-27 & 5248.5 & 4255.6 & 992.96 & 4752.04 & 4700.17 & 4.15 & 4726.03 & 1.67 & 1.16 & 0.81 \\
\hline $\begin{array}{c}\text { Check } \\
\text { (Pirsabak- } \\
\text { 2008) }\end{array}$ & 5644.1 & 5000.0 & 644.07 & 5322.04 & 5302.55 & 2.51 & 5312.28 & 2.11 & 1.37 & 0.89 \\
\hline
\end{tabular}




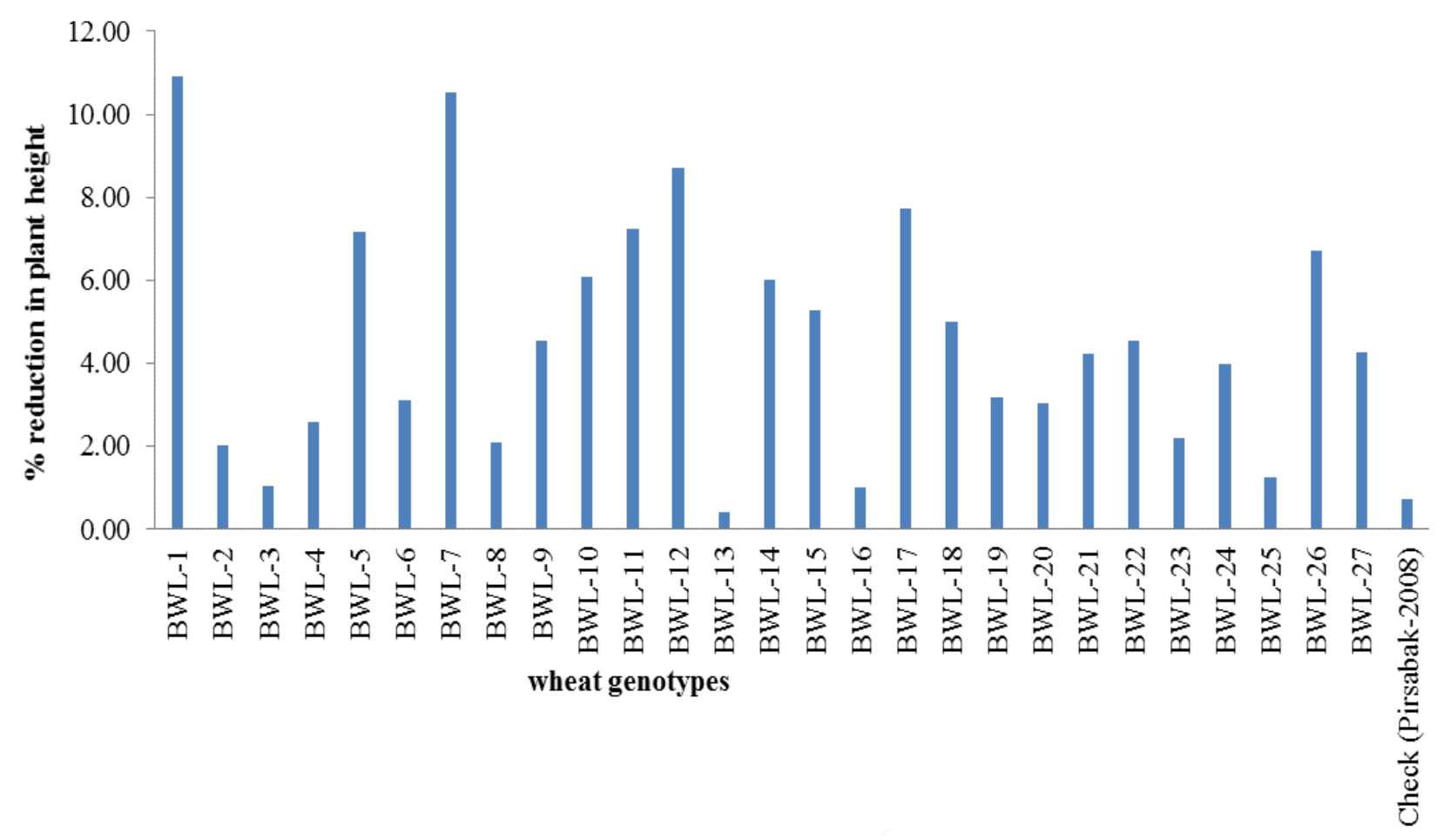

Figure 1. \% reduction in plant height of wheat genotypes under late planting as compared to normal (optimum) planting conditions

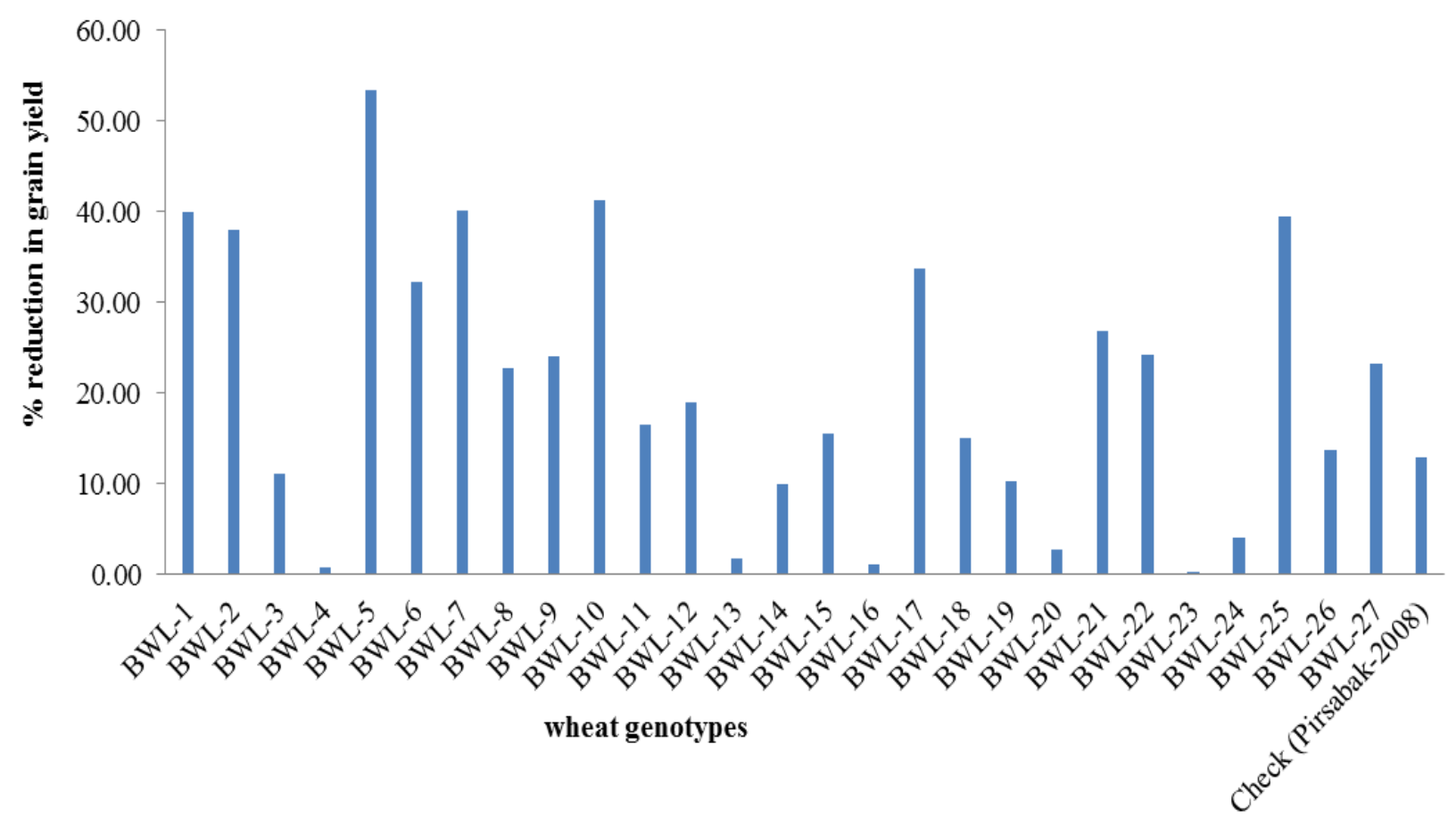

Figure 2. \% reduction in grain yield of wheat genotypes under late planting as compared to normal (optimum) planting conditions 
Table 5. Correlation coefficient of plant height and grain yield under normal and late planting (stress) with various selection indices

\begin{tabular}{|c|c|c|c|c|}
\hline & \multicolumn{2}{|c|}{ Plant height } & \multicolumn{2}{c|}{ Grain yield } \\
\hline Selection Indices & Normal Planting & Late planting & Normal Planting & Late planting \\
\hline GM & $0.96^{* *}$ & $0.94^{* *}$ & $0.92^{* *}$ & $0.94^{* *}$ \\
\hline TOL & $0.55^{* *}$ & -0.05 & $0.46^{* *}$ & $-0.28^{\mathrm{NS}}$ \\
\hline MP & $0.96^{* *}$ & $0.94^{* *}$ & $0.93^{* *}$ & $0.92^{* *}$ \\
\hline HM & $0.95^{* *}$ & $0.95^{* *}$ & $0.91^{* *}$ & $0.95^{* *}$ \\
\hline SSI & $0.48^{* *}$ & $-0.13^{\mathrm{NS}}$ & $0.21^{\mathrm{NS}}$ & $-0.45^{* *}$ \\
\hline STI & $0.96^{* *}$ & $0.94^{* *}$ & 0.91 & $0.94^{* *}$ \\
\hline YSI & $-0.46^{* *}$ & $0.14^{\mathrm{NS}}$ & $-0.29^{\mathrm{NS}}$ & $0.44^{* *}$ \\
\hline YI & $0.81^{* *}$ & $1.00^{* *}$ & $0.72^{* *}$ & $1.00^{* *}$ \\
\hline
\end{tabular}

$*$ and $* *=$ Significant at $5 \%$ and $1 \%$ level of probability respectively NS = Non-significant

\section{Conclusions and recommendation}

Analyzed data revealed highly significant differences among the genotypes evaluated under normal and late planting condition indicating that genotypes were of diverse genetic background. Based on grain yield, among the genotypes, Pirsabak-2008, BWL-12 and BWL23 were found best under both non-stress (normal) and stress (late) planting environments. Reduction in plant height and grain yield was observed when genotypes were planted late as compared to normal (optimal) conditions. Stress selection indices are acceptable tools and have been widely used in identification of genotypes with desirable yield performance under both normal and late planting conditions. Based on Mean productivity (MP), Geometric mean productivity (GMP), Harmonic mean (HM), Stress tolerance Index (STI) and Yield Index (YI), Pirsabak-2008, BWL-23 and BWL-27 were relatively promising and late planting stress tolerant genotypes and could be sown in both normal and late planting environments. Under normal and late planting conditions, significant positive correlation of plant height and grain yield with stress selection indices (GM, HM, SSI and YI) indicated that these indices are effective in identification of lines/ genotypes under different planting conditions.

\section{Authors' contributions}

Conceived and designed the experiments: $\mathrm{M}$ Ishaq \& G Ahmad, Performed the experiments: IA Shah, N Ahmad \& A Saleem, Analyzed the data: M Ali \& M Khalid, Contributed materials/ analysis/ tools: TU Khan, B Iqbal, B Ahmad,
MA Qureshi, M Miraj \& K Din, Wrote the paper: M Ishaq \& K Afridi.

\section{Acknowledgments}

The authors are thankful to CIMMYT (International Maize and Wheat Improvement Center-Mexico) for the provision of germplasm and Wheat productivity Enhancement Program (WPEP) Pakistan for the financial support.

\section{References}

1. Khalil HI, Wahab A, Nayab D, Ghani SS \& Ullah H (2016). Heritability and selection response for morphological and yield traits in normal and late planted wheat. $A d v$ Environ Biol 10(9): 172-179.

2. Hussain A, Maqsood M, Ahmad A, Aftab S \& Ahmad Z (1998). Effect of irrigation during various development stages on yield, component of yield and harvest index of different wheat cultivars. Pak J Agric Sci 34: 104-107.

3. Nouri A, Etminan A, Teixeira da Silva JA \& Mohammadi R (2011). Assessment of yield, yield-related traits and drought tolerance of durum wheat genotypes (Triticum turgidum var. durum Desf.). Aust J Crop Sci 5(1): 816.

4. Clarke JM, Towenley-Smith TM, McCaig TND \& Green G (1984). Growth analysis of spring wheat cultivars of varying drought resistance. Crop Sci 24: 537-541.

5. Khan FU \& Mohammad F (2016). Application of stress selection indices for assessment of nitrogen tolerance in wheat (Triticum aestivum L.) J Anim Plant Sci 26(1): 201-210. 
6. Fischer RA \& Maurer R. (1978). Drought resistance in spring wheat cultivars. I. Grain yield responses. Aust J Agric Res 29: 897912.

7. Fernández GCJ (1992). Effective Selection Criteria for Assessing Plant Stress Tolerance. In: Proceedings of the International Symposium on Adaptation of Vegetables and Other Food Crops in Temperature and Water Stress (Kuo CG, ed.). Publication, Tainan, Taiwan.

8. Gavuzzi P, Rizza F, Palumbo M, Campaline RG, Ricciardi GL \& Borghi B (1997). Evaluation of field and laboratory predictors of drought and heat tolerance in winter cereals. Can J Plant Sci 77: 523-531.

9. Bouslama M \& Schapaugh WT (1984). Stress tolerance in soybean. Part 1: Evaluation of three screening techniques for heat and drought tolerance. Crop Sci 24: 933-937.

10. Annicchiarico P (2002). Genotype $\times$ environment interaction: challenges and opportunities for plant breeding and cultivar recommendations. FAO, Rome. Plant Production and Protection pp: 174.

11. Rosielle AA \& Hamblin J (1981) Theoretical aspects of selection for yield in stress and non-stress environments. Crop Sci 21: 943- 945.

12. Kristin AS, Senra RR, Perez FI, Enriquez BC, Gallegos JAA, Vallego PR, Wassimi N \& Kelley JD (1997). Improving common bean performance under drought stress. Crop Sci 37: 43-50.

13. Talebi R, Fayaz F \& Naji AM (2009). Effective selection criteria for assessing drought stress tolerance in durum wheat (Triticum durum Desf.). General and Appl Plant Phys 35(1-2): 64-74.

14. Ahmad F, Khan S, Ahmad SQ, Khan H \& Muhammad F (2011). Genetic analysis of some quantitative traits in bread wheat across environments. Afric J Agric Res 6(3): 686-692.

15. Gul H, Saeed B, Khan AZ, Latif U, Ali K, Rehman JU \& Rehman SU (2012). Yield and yield contributing traits of wheat cultivars relation with planting dates and nitrogen fertilization. J Agric Bio Sci 7(6): 1990-6145.

16. Inamullah $\mathrm{A}$, Shah $\mathrm{H}, \mathrm{Haq} \mathrm{Z}$ \& Khan $\mathrm{FU}$ (2007). An analysis of the planting dates effect on yield and yield attributes of spring wheat. Sarhad J Agric 23(2): 1530-1535.

17. Baloch MS, Nadimi MA, Zubair M, Awan I, Khan EA \& Ali S (2012). Evaluation of wheat under normal and late sowing conditions. Pak J Bot 44(5): 1727-1732.

18. Irfaq M, Muhammad T, Amin M \& Jabbar A (2005) Performance of yield and other agronomic characters of four wheat genotypes under heat stress. Pak J Bot 1(2): 124-127.

19. Subhan F, Nazir A, Anwar M, Nazir HS, Siddiq M, Rahman J \& Sajjad T (2004). Response of newly developed wheat cultivars/advance lines to planting dates in the central agro-ecological zone of NWFP. Asian J of Plant Sci 3(1): 87-90.

20. Najafian G (2009). Drought tolerance indices, their relationships and manner of application to wheat breeding programs. In: Mohammadi R, Hagh Parast R (Eds.) Plant Science in Iran. Middle Eastern Russian J Plant Sci Biotech 3(SI1): 25-34.

21. Farshadfar E, Ghannadha M, Zahravi M \& Sutka J (2001). Genetic analysis of drought tolerance in wheat. Plant Breed 114: 542544.

22. Dehghani GH, Malekshhi F \& Alizadeh B (2009). A study of drought tolerance indices in canola (Brassica napus L.) genotypes. $J$ Sci \& Tech Agric \& Natural Resources 13(48): 77-90.

23. Chio BH, Park KY \& Park RK (1992). A study of cultural methods for summer Buck wheat sown in spring. Korean J Crop Sci 37: 149-154.

24. Pecio A \& Wielgo B (1999). Buck wheat yielding and structure of plant and canopy dependent of planting time. J Agric 16: 5-17. 